up and down. Difficult conditions exist more in advanced levels than in primary and secondary-school levels. For example, the best observatory admits the public but hardly allows cooperation in research with other universities. The government ordinarily pays little attention to astronomical support since such support is not in the country's development plans. Many astronomers, lacking sufficient equipment and encouragement, often find it so difficult to carry out their research that they turn their attention to other fields. Moreover, advanced journals and textbooks are insufficient. All these cause gaps in astronomy education.

These problems may be eradicated by cooperation with astronomers from welldeveloped countries in astronomy. It would be good to establish well-planned observatories in Thailand, managed by an efficient staff of foreign astronomers who could make justified allowances to enable enthusiastic Thai astronomers to participate in research. Starting from such a step, Thai astronomy would be likely to be on the threshold of prosperity.

\title{
THE TEACHING OF ASTRONOMY AND SOCIAL AND ECONOMIC DEVELOPMENT
}

\author{
Gonzalo Vicino
}

A.N.E.P., Consejo de Educación Secundaria, Uruguay

Sometimes we may ask ourselves how can astronomy, a science with apparently few points of contact with technology or industrial production, help the economic development of people?

My paper is an account of an experiment undertaken in Uruguay on the teaching of astronomy in secondary schools. We seek to show how astronomy may contribute to the development of nations that, like Uruguay, need to create their own technologies to overcome their economic backwardness.

Though the introduction of astronomy in the secondary education curriculum in Uruguay dates back to 1889 , in 1986 the Educational Supervisory Office made a radically different proposal for the methodological and programmatic orientation of astronomy teaching. The proposed goal is clear: the teaching of astronomy in secondary education should be geared not to train technicians but to create a scientific-minded youth. It is not important if later, in their university studies, these young people go into medicine, engineering, or economics; the important thing is a reevaluation of sciences in the eyes of adolescents, who often slide along the comfortable slope of certain humanistic areas, in which they wind up by the process of elimination. (One often hears the argument, "I'm studying law because it doesn't have mathematics.") Further, above all those considerations, the most important thing is to create thinking minds, the minds of free people. 
We firmly believe that astronomy is the integrating science par excellence, in which adolescent students can find clear bonds with almost all the branches of knowledge, including exact and natural sciences as well as social sciences, philosophy, and the arts. It is this integrating vision of astronomy that makes it the door leading youth along the way to the sciences.

But how can a subject be made attractive enough to draw young minds towards the sciences? We propose the concept of "active learning" in place of the classical "passive learning," in which the pupils receive the knowledge from their teachers. In our concept, teachers must not "show" the students the facts: they must show them the way to discover facts by themselves. Education cannot be imposed: nobody educates anyone else, but only himself; adolescents must be the protagonists of their own discovery adventure. Likewise, we must recover the cheerful sense of study, of discovery achieved through a healthy and vital curiosity. Moreover, the study must have a close relation with daily life; the abstraction capacity of young people, to a great extent reduced by the so-called "image culture" in which we live, cannot be developed overnight, or by mere imposition. Therefore, as a starting point for science studies, we must inculcate the habit and pleasure of the observation of nature.

To be able to adequately develop this observational stage, before analyzing and discussing phenomena, the students must learn to build their own elementary instruments, for instance an "astronomical crossbow" to measure angular distances, or a simple spectroscope with which to observe light dispersion. This activity thus stimulates the adolescent's manual ability and creativity, and at the same time involves many other students, perhaps intellectually less able but manually skilled, who otherwise must be left out in an eminently theoretical course.

From the very beginning of the course, we seek to have our pupils learn to observe (to measure) several phenomena with which data they will have to reach some conclusions some 5 or 6 months later; these observations must be ordered and recorded in such a way that students can learn to use them in a statistical way, and then deduce the regularities that lead to identification of natural laws. These systematic observations can be, for example, about the sun's visibility, the movements of the moon and planets, and so on.

But while they record these data, in the classroom the course is developed with the treatment and discussion of such subjects as the magnitude scale, the origin and denomination of constellations, the chemical composition of stars (presenting a wide view about spectral analysis and matter's atomic structure), the stars' surface temperatures (including experiments about radiation laws), always seeking to relate each discovery in a deductive way with the subsequent ones.

I am not going to describe all the program, but I can mention the remaining subjects: "The Universe," "Life in the Universe" (which implies a study of the solar system), and "The Steps of Astronomy" (a historical vision involving the discovery of the causes of seasons, the moon's movements, the geocentric and heliocentric systems, and so on). The historical view of the last of these leads us through Greek and Arabic astronomy, up to the Renaissance through the great developments made 
by Kepler, Galileo, and Newton, and through the development of modern astronomy.

For many years, our country has suffered from a strong propensity in university studies toward law and humanities over all other disciplines: on the average, 30 per cent of students go to law school, and together with humanities students the total is near to 50 per cent; meanwhile, we find some 5 to 10 per cent in engineering, 3 to 4 per cent in chemistry, 3 to 5 per cent in architecture, and only 2 to 3 per cent in agronomy.

It is still too early, only two years after the implementation of this new astronomy methodology, to show statistical data to prove the influence of this renewed approach on vocational orientation. Perhaps it may be necessary to wait 10 to 15 years to see the evidence of that influence. But there are some very positive indications that let us affirm we are in the right path: during the first two years with the new astronomy curriculum, many astronomy clubs have sprung up around the whole country. We cannot be absolutely certain about the future of these young people, but we have evidence of their interest, their yearning for scientific knowledge. They are our hope; they are the ones who will be able to change the ratio of students in scientific vs. humanistic disciplines. And this is probably where the future economic and social development of our country comes into play.

\title{
PROGRAMS OF I.A.U. COMMISSION 46 FOR DEVELOPING COUNTRIES
}

\author{
Donat G. Wentzel \\ University of Maryland, College Park, Maryland 20742, U.S.A.
}

\section{International Schools for Young Astronomers (ISYA)}

ISYA typically last about three weeks and attract roughly twenty students at the Master of Science level. About half the students are from the host country, the others from neighboring countries. ISYA are initiated by astronomically developing countries and have been held in Venezuela, Nigeria, Egypt, Portugal, Yugoslavia, Indonesia, China, and others. Two or three central topics are chosen. For each topic, a suitable faculty member is invited to stay for the entire school. Other topics appear for enrichment and are chosen according to local expertise and availability of other visitors. The IAU provides the prestige of an international venture and seeks to garner local support not only for the ISYA but also for the longer-term increase in local astronomical capabilities. Financial support is provided both locally and by the IAU, when possible ultimately from UNESCO. Perhaps the greatest benefits of an ISYA are the resulting lasting acquaintances and scientific cooperations. 